\title{
REVISTAMATËRIA
}

\section{Análise não linear de chapas através de uma formulação do método dos elementos de contorno com convergência quadrática}

\section{The stretched plate problem analyzed by a boundary element formulation with quadratic convergence}

Vinicio da Cunha Dóro ${ }^{1}$, Gabriela Rezende Fernandes ${ }^{2}$

\author{
${ }^{1}$ FEIS- Faculdade de Engenharia de Ilha Solteira CEP: 15385-000- (18)3743-1000, Ilha Solteira, SP \\ e-mail: viniciodoro@gmail.com \\ 2 e-mail: gabrielar.fernandes@gmail.com
}

\section{RESUMO}

No presente trabalho foi desenvolvida a formulação não-linear do método dos elementos de contorno para a análise estrutural de chapas escrita em termos de deslocamentos e forças nas direções normal e tangencial ao contorno da sua superfície. A equação integral do deslocamento é deduzida a partir do Teorema de Reciprocidade de Betti, considerando-se espessura constante na chapa. Para calcular a integral de domínio envolvendo o campo de esforços iniciais (ou inelásticos) deve-se discretizar o domínio em células. A solução não linear se obtém por uma formulação implícita, na qual as correções das deformações são feitas através do operador tangente consistente que se atualiza a cada nova iteração, tendo como referência os valores das variáveis internas referentes ao incremento convergido, o que leva a uma convergência quadrática do processo iterativo. Utilizou-se como critério de ruptura o de von Misses e exemplos foram analisados a fim de mostrar a convergência quadrática no processo iterativo e também a convergência dos resultados numéricos a medida que se refinava a discretização do contorno em elementos e do domínio em células.

Palavras-chave: método dos elementos de contorno, operador tangente consistente, análises não lineares.

\section{ABSTRACT}

In this paper the non-linear formulation of the boundary element method (BEM) for analyzing the stretching plate problem written in terms of displacements and tractions in the normal and tangential directions to the boundary has been developed. The integral equation of displacement is derived from Betti's reciprocity theorem, considering constant thickness on the plate. To obtain the non-linear solution an initial (or inelastic) force field must be considered over the plate domain, requiring therefore the plate domain discretization into cells. Besides, an implicit formulation is adopted, where the strains correction to be computed for each iteration is obtained by considering the consistent tangent operator, leading to a quadratic convergence rate in the iterative procedure required to achieve the plate equilibrium. In the numerical examples the results are compared to software ANSYS and the Von Mises criterion has been adopted to model the material behavior, showing the quadratic convergence rate. Besides, different discratizations have been analyzed in order to show as well the results convergence.

Keywords: boundary element method, consistent tangent operator, non-linear analysis.

\section{INTRODUÇÃO}

Problemas de engenharia simulados numericamente é uma realidade em análises estruturais, de forma que cada vez mais o avanço tecnológico dos hardwares e softwares dá subsidio a simulações mais complexas. Os métodos mais utilizados para a representação numérica a partir de problemas físicos são: o método dos 
elementos finitos (MEF), o método das diferenças finitas (MDF) e o mais recente entre eles, o método dos elementos de contorno (MEC) introduzido por [1].

A utilização do método dos elementos de contorno apresenta algumas particularidades sendo:

- a possibilidade da redução da dimensionalidade do problema, ocasionando uma menor quantidade de dados de entrada e assim, utilizando menos memória computacional;

- os deslocamentos e forças são aproximados no elemento através da mesma função de interpolação, ou seja, eles têm a mesma ordem de aproximação;

- em casos de concentração de tensões apresenta bons resultados;

- em caso onde há interação da estrutura com o meio infinito, pois não há necessidade de discretização do meio infinito, apenas o contorno da estrutura.

A formulação não-linear do MEC apresentada nesse trabalho foi desenvolvida por FERNANDES [8], tendo sido obtida através da inclusão de um campo de forças normais iniciais na formulação linear do MEC para análise linear de chapas. Para aproximar esses esforços normais iniciais no domínio, este deve ser discretizado em células triangulares (ver mais detalhes em [9] e [10]), como demonstrado na figura 4, onde se adotam funções de aproximação lineares para esses esforços. Portanto, para obtenção da solução não-linear, deve-se discretizar o contorno em elementos e o domínio em células. Diversos trabalhos já publicados tratam de análise de placas através do MEC, podendo citar [2], [3] [5], [7], [8], [11] e [14]. Alguns desses trabalhos tratam sobre o problema de flexão de placas, outros sobre o problema bidimensional ou do acoplamento entre esses dois problemas, a fim de se fazer análise de flexão composta de placas. Nesse trabalho, a solução é obtida a partir da formulação implícita, na qual as correções que devem ser dadas aos estados de deformações planas em uma determinada iteração, são obtidas através do operador tangente consistente discutido em [13], que é atualizado a cada iteração, e do resíduo de esforços.

A seguir, inicialmente mostram-se as equações integrais e algébricas do MEC para análise nãolinear do problema bidimensional. Posteriormente, deduz-se a equação de resíduos (ou equação de equilíbrio) em termos de esforços normais verdadeiros $\left(N_{n}\right)$ e deformações planas $(\varepsilon)$, que deve ser satisfeita no processo iterativo para que a placa alcance seu equilíbrio. Aplicando-se o Método de Newton Raphson, obtêm-se a solução linearizada do problema, onde as correções que devem ser dadas às deformações na próxima iteração são calculadas a partir da matriz tangente consistente, o que gera taxa de convergência quadrática do erro no processo iterativo. Então, mostra-se o processo incremental-iterativo no qual a atualização do tensor constitutivo, das tensões e das variáveis internas relacionadas ao modelo constitutivo é feita tendo como referência o incremento anterior convergido.

\subsection{Objetivo}

O objetivo deste trabalho é desenvolver a formulação não linear do método dos elementos de contorno aplicado a chapas apresentada em [8], onde se utiliza o operador tangente consistente no processo iterativo, o que leva a uma taxa de convergência quadrática do erro. São também analisados exemplos numéricos onde se mostra a convergência dos resultados à medida que se refina a discretização do contorno em elementos e do domínio em células. Além disso, nesses exemplos é apresentada também a convergência quadrática do erro ao longo do processo iterativo referente a determinados incrementos de carga.

\section{2 - EQUAÇÕES BÁSICAS DO PROBLEMA NÃO-LINEAR DE CHAPAS}

Considerando uma chapa qualquer de espessura $h$, domínio $\Omega$ como mostrado na figura 1 e com carregamento somente nos eixos $x 1$ e $x 2$ tem-se que o equilíbrio de um ponto na chapa é dado pela equação (1).

$$
\dot{N}_{i j, j}+\dot{b}_{i}=0 \quad \mathrm{i}, \mathrm{j}=1,2
$$

onde $\dot{b}_{i}$ representa as forcas distribuídas no plano da chapa e $\dot{N}_{i j, j}$ a derivada da força normal na chapa 


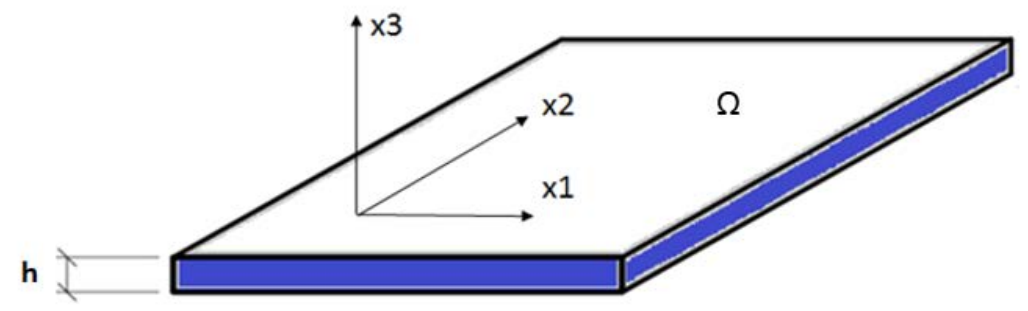

Figura 1: Elemento representativo para o problema de chapa

Na análise não-linear, a deformação total é dividida em uma parcela elástica e uma parcela plástica (ou residual) e os valores da tensão são calculados por um modelo constitutivo. Nesse trabalho, será adotado um modelo elasto-plástico com encruamento isótropo, como descrito na figura 2 para o caso uniaxial. No entanto, a formulação apresentada nesse trabalho pode ser usada com qualquer tipo de modelo constitutivo.

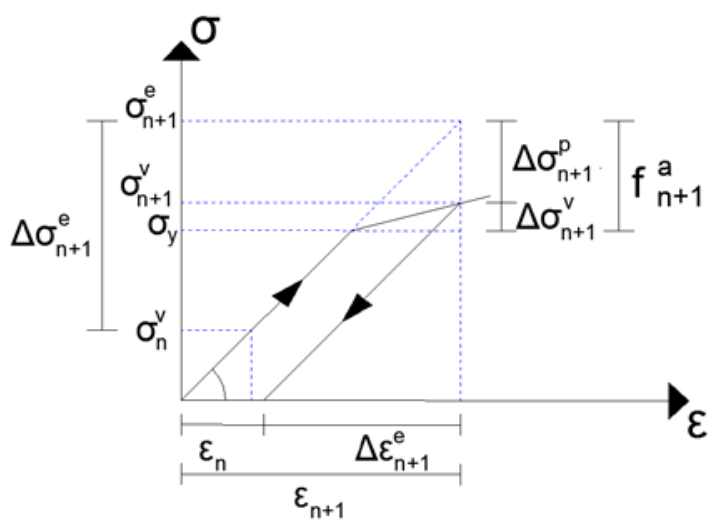

Figura 2: Correção da tensão no modelo elasto-plástico uniaxial com encruamento isótropo positivo

onde: $\Delta \sigma^{e}$ é o incremento de tensão de tentativa; $\sigma^{e}$ é a tensão total de tentativa; $\sigma^{v}$ é a tensão verdadeira; $\Delta \sigma^{p}$ é a parcela plástica de $\Delta \sigma^{e}$, ou o excesso de tensão; $\Delta \sigma^{v}$ é o incremento verdadeiro de tensão; $f_{t+\Delta t}^{a}$ é o valor do critério no início da iteração correspondente ao instante $t+\Delta t$.

Adicionando o incremento de tensão de tentativa à tensão do incremento anterior, tem-se a tensão total de tentativa que será usada para verificar o modelo constitutivo. Então, de acordo com o modelo constitutivo adotado, calculam-se a tensão verdadeira $\left(\sigma^{v}\right)$ e o tensor constitutivo elasto-plástico. Note que a tensão verdadeira se relaciona com a deformação elástica via lei de Hooke, mas se relaciona com a deformação total via tensor constitutivo elasto-plástico.

Assim as tensões elásticas (ou de tentativa), se relacionam com as deformações totais através da lei de Hooke, podendo ser escritas como soma das tensões verdadeiras com as tensões plásticas. Na forma de taxa, as tensões de tentativa podem ser escritas como:

$$
\dot{\sigma}^{e}=\dot{\sigma}+\dot{\sigma}^{p}
$$

Analogamente, as taxas das forças normais elásticas $\dot{N}_{i j}^{e}$ (ou forças normais de tentativa) são dadas por:

$$
\dot{N}_{i j}^{e}=\dot{N}_{i j}^{p}+\dot{N}_{i j} \quad \quad \mathrm{i}, \mathrm{j}=1,2
$$

onde: $\dot{N}_{i j}^{e}$ são taxas das forças normais elásticas; $\dot{N}_{i j}$ as taxas das forças normais verdadeiras (obtidas a partir das tensões verdadeiras); $\dot{N}_{i j}^{p}$ as taxas das forças normais plásticas.

A partir da equação de Navier, que representa a equação de equilíbrio para o caso linear, chega-se à equação diferencial do estado plano para análises não lineares: 


$$
\dot{u}_{i, j j}+\frac{1}{\left(1-2 v^{\prime}\right)} \dot{u}_{j, i j}+\frac{\left(b_{i}-\dot{N}_{i j, j}^{e}\right)}{\bar{G}}
$$

em que :

$$
v^{\prime}=\frac{v}{1+v}
$$

As relações básicas são completadas considerando-se as condições de contorno. Então, divide-se o contorno total em duas partes $\Gamma_{1}$ e $\Gamma_{2}$ ou seja faz-se: $\Gamma=\Gamma_{1}+\Gamma_{2}$. Admitindo que em $\Gamma_{1}$ se prescreva o deslocamento $u_{i}$ e em $\Gamma_{2}$ a tensão $p_{i}$, tem-se: $u_{i}=\bar{u}_{i}$ em $\Gamma_{1}$ ( condição de contorno essencial), $p_{i}=\bar{p}_{i}$ em $\Gamma_{2}$ (condição de contorno natural).

\subsection{Equação integral do deslocamento}

A equação integral do deslocamento no plano da chapa $u_{i}$ é obtida a partir do teorema de Betti, integrando ao longo da espessura da chapa $(h)$ (ver figura1), ou seja:

$$
\int_{\Omega} \varepsilon_{i j k}^{*} \dot{N}_{j k}^{e} d \Omega=\int_{\Omega} \dot{\varepsilon}_{j k} \dot{N}_{i j k}^{*} d \Omega \quad i, \mathrm{j}, \mathrm{k}=1,2
$$

onde os termos com * representam o problema fundamental

Considerando a relação (3), pode-se reescrever a equação (6):

$$
\int_{\Omega_{m}} \varepsilon_{i j k}^{*} \dot{N}_{j k} d \Omega=\int_{\Omega_{m}} \dot{\varepsilon}_{j k} \dot{N}_{i j k}^{*} d \Omega-\int_{\Omega_{m}} \varepsilon_{i j k}^{*} \dot{N}_{j k}^{p} d \Omega
$$

Integrando-se a equação (7) por partes, obtém-se a representação integral (exata) do deslocamento para pontos de colocação internos. A partir dessa, pode-se obter a equação integral para pontos sobre o contorno, onde surge o termo livre $K_{u i}$. De forma genérica, pode-se escrever a equação integral exata da taxa do deslocamento na direção $i\left(\dot{u}_{i}\right)$ como:

$$
\mathrm{K}_{u i} \dot{u}_{i}=-\int_{\Gamma}\left[\dot{u}_{n} p_{i n}^{*}+\dot{u}_{s} p_{i s}^{*}\right] d \Gamma+\int_{\Gamma}\left[\dot{p}_{n} u_{i n}^{*}+\dot{p}_{s} u_{i s}^{*}\right] d \Gamma+\int_{\Omega}\left[u_{i n}^{*} \dot{b}_{n}+u_{i s}^{*} \dot{b}_{s}\right] d \Omega+\int_{\Omega} \varepsilon_{i j k}^{*} \dot{N}_{j k}^{p} d \Omega
$$

onde: $\Omega$ é a área carregada da chapa; o termo livre $K_{u i}$ pode assumir vários valores dependendo da posição do ponto de colocação como segue: $K_{u i}=1$ e $K_{u i}=1 / 2$, respectivamente, para pontos internos e pontos no contorno não coincidentes com um canto.

Este trabalho lida com análises elastoplásticas, requerendo então o uso de um processo incremental iterativo. Assim, todos os valores escritos na forma de taxas devem ser transformados em incrementos. Considerando-se o passo de tempo $\Delta t=t_{n+1}-t_{n}$ como um passo típico de tempo, a solução do problema consiste em procurar a solução no passo de tempo $t_{n+1}$ quando se conhece a solução no passo $t_{n}$. O incremento de uma variável ( $\Delta a$ ) é dado por:

$$
\Delta a=a_{n+1}-a_{n}=\Delta t \dot{a}_{n+1}
$$

sendo $\dot{a}_{n+1}=\frac{\Delta a}{\Delta t} ; \dot{a}_{n+1}=\frac{d\left(a_{n+1}\right)}{d t}$

Note que para escrever as equações integrais na forma incremental, não há a necessidade de reescrever as integrais já deduzidas, basta substituir os valores das taxas pelos incrementos correspondentes.

Note que têm-se duas incógnitas em cada nó de contorno, além de três componentes de forças iniciais em cada nó de célula. Assim, para obter a solução do problema serão consideradas as equações integrais dos deslocamentos escritas nos nós de contorno e as equações dos esforços elásticos escritas nos nós de células. Após resolver o processo iterativo em um dado incremento, as incógnitas no contorno, assim 
como os valores das forças iniciais nos nós de células são conhecidos. Então os deslocamentos e tensões em um ponto interno qualquer podem ser obtidos escrevendo a respectiva equação algébrica. A equação que representa a rotação da chapa é dada a partir da diferenciação da equação (8):

$$
\begin{aligned}
& \Delta u_{i}, \ell=-\int_{\Gamma_{m}}\left(\Delta u_{n} p_{i n, \ell}^{*}+\Delta u_{s} p_{i s}^{*}, \ell\right) d \Gamma+\int_{\Gamma}\left(u_{i n, \ell}^{*} \Delta p_{n}+u_{i s}^{*}, \ell \Delta p_{s}\right) d \Gamma+ \\
& +\int_{\Omega_{b}}\left(u_{i n, \ell}^{*} \Delta b_{n}+u_{i s}^{*}, \ell \Delta b_{s}\right) d \Omega++\frac{\partial}{\partial x_{l}} \int_{\Omega} \varepsilon_{i j k}^{*} \Delta N_{j k}^{p} d \Omega+\int_{\Gamma}\left(u_{i n, \ell}^{*} \Delta p_{n}+u_{i s}^{*}, \ell \Delta p_{s}\right) d \Gamma
\end{aligned}
$$

A integral de domínio envolvendo a força normal plástica $\left(\Delta N_{j k}^{P}\right)$ apresenta singularidades quando o ponto de colocação pertence à célula a ser integrada. Então para obter-se o incremento do deslocamento tem-se que eliminar essa singularidade. Para isso, retira-se do domínio $\Omega$ um domínio circular $\Omega_{c}$, de pequeno raio $\varepsilon$ e com origem no ponto $q$, obtendo-se o domínio $\Omega_{\varepsilon}$, que é dado por: $\Omega_{\varepsilon}=\Omega-\Omega_{c}$ como visto na figura 3.

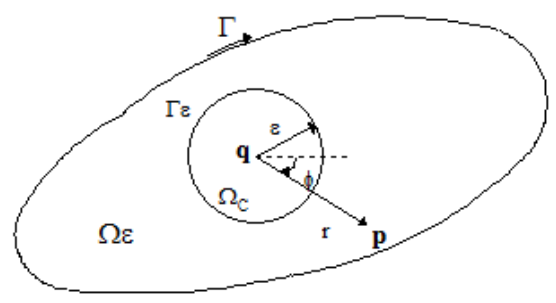

Figura 3: Domínio $\Omega_{\varepsilon}$ e $\Omega_{c}$ Fonte: Fernandes, 2003

Então a integral de domínio da equação da derivada direcional de $u_{i}$, definida em (10), pode ser escrita como:

$$
I=\frac{\partial}{\partial x_{1}} \int_{\Omega} \varepsilon_{i j k}^{*} \Delta N_{j k}^{p} d \Omega=\frac{1}{16 \mu\left(1-v^{\prime}\right)}\left[\left(6-8 v^{\prime}\right) \Delta N_{i l}^{p}+\Delta N_{m m}^{p}(q) \delta_{k l}\right] \quad \quad \mathrm{i}, \mathrm{j}, \mathrm{k}, \mathrm{l}, \mathrm{m}=1,2
$$

Para calcular as integrais de domínio das equações (8) e (10) que envolvem os esforços plásticos, deve-se discretizar o domínio em células, nas quais as forças normais iniciais de um ponto $p$ da chapa serão aproximadas nos nós por funções interpoladoras. Neste trabalho foram adotadas células triangulares, como representado na figura 4 (ver mais detalhes em [4]), com funções de aproximação lineares.

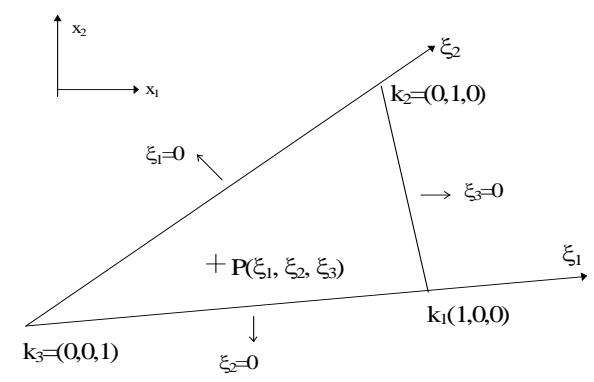

Figura 4: Célula triangular com coordenadas homogêneas

\section{2 - Equações algébricas do MEC para análise não-linear}

As equações integrais apresentadas até então, podem ser transformadas em equações algébricas, discretizando o contorno em elementos e o domínio em células, nos quais as variáveis de um ponto $p$ qualquer são escritas em função dos seus valores nodais, utilizando-se as funções de interpolação. 
Após a integração numérica ao longo de todos os elementos do contorno e de todas as células, as equações de deslocamentos referentes aos problemas de chapa (equação de $u_{k}$ ou $u_{k}$, ) de um ponto interno ou sobre o contorno externo, podem ser escritas, em suas formas matriciais. Escrevendo-se então as equações dos deslocamentos $u_{s}$ e $u_{n}$ em cada nó do contorno, obtém-se o seguinte sistema de equações:

$$
[H]\{\Delta U\}=[G]\{\Delta P\}+\{\Delta T\}+[E]\left\{\Delta N^{P}\right\}
$$

onde: $\{\Delta U\}$ é o vetor dos incrementos dos deslocamentos no contorno; $\{\Delta P\}$ é o vetor dos incrementos das forças no contorno; $\{\Delta T\}$ é o vetor da carga externa; $[E]$ é a matriz obtida integrando as células (Figura 4); $\left\{\Delta N^{P}\right\}$ é vetor do incremento de forças plásticas.[H] é resultado da integração dos esforços fundamentais ao longo do contorno; $G]$ é o resultado da integração dos deslocamentos fundamentais ao longo do contorno.

A equação (12) pode ser representada da seguinte forma:

$$
\underset{\sim}{H} \Delta \underset{\sim}{U}=\underset{\sim}{G} \underset{\sim}{P}+\Delta \underset{\sim}{T}+\underset{\sim}{E} \Delta \underset{\sim}{P}
$$

Para a resolução numérica da análise não linear, são feitas operações matriciais sobre a equação (13), visando-se deixar os termos referentes aos esforços iniciais ou plásticos isolados. Assim, armazenam-se todas as incógnitas do contorno externo e cantos num vetor $\{X\}$, trocando-se as respectivas colunas entre as matrizes $[H]$ e $[G]$. Então, somam-se em $\{B\}$ os efeitos dos deslocamentos ou esforços prescritos no contorno externo, ao efeito do carregamento que atua na chapa ,obtendo-se:

$$
\underset{\sim}{A} \Delta \underset{\sim}{X}=\underset{\sim}{B}+\underset{\sim}{E} \Delta \underset{\sim}{N}
$$

sendo:

$$
\underset{\sim}{\Delta B}=\underset{\sim}{G} \underset{\sim}{\Delta} P+\underset{\sim}{\Delta T}
$$

Multiplicando-se os dois lados dessa equação por $[A]^{-1}$, obtêm-se as incógnitas do problema:

$$
\Delta \underset{\sim}{X}=\underset{\sim}{L}+\underset{\sim}{R_{N}} \Delta N_{\sim}^{P}
$$

sendo:

$$
\begin{aligned}
& \underset{\sim}{L}=A^{-1} \underset{\sim}{B} \\
& {\underset{\sim}{N}}_{N}={\underset{\sim}{A}}^{-1}{\underset{\sim}{E} S}_{S}
\end{aligned}
$$

Assim, o termo $R_{N}$ representa a influência das forças normais plásticas nos valores dos deslocamentos do contorno e $[L]$ a resposta elástica sem considerar os esforços iniciais ou plásticos. No caso da análise não linear onde a chapa é sujeita a esforços iniciais, os deslocamentos e esforços finais são obtidos somando às incógnitas da análise linear, os valores devido aos esforços iniciais. Os resultados finais são obtidos após se fazer o processo incremental-iterativo onde, no final de um incremento, o vetor dos esforços iniciais ou plásticos corresponde aos valores obtidos na iteração onde o equilíbrio foi alcançado.

Analogamente se faz para os pontos internos, utilizando-se o vetor $[L]$ calcula-se o deslocamento elástico devido ao carregamento, então se acrescenta ao valor desse, a parcela referente aos esforços iniciais:

$$
\begin{aligned}
& \underset{\sim}{\Delta U}(q)=\underset{\sim}{H} \Delta \underset{\sim}{U}+\underset{\sim}{G} \underset{\sim}{\Delta P}+\underset{\sim}{\Delta T}+\underset{\sim}{E_{N}} \underset{\sim}{\Delta N^{P}} \\
& \underset{\sim}{U}=\underset{\sim}{A} \underset{\sim}{\Delta X}+\underset{\sim}{\Delta B}+\underset{\sim}{E_{N}} \underset{\sim}{\Delta N^{P}}
\end{aligned}
$$

Substituindo (16) em (19): 


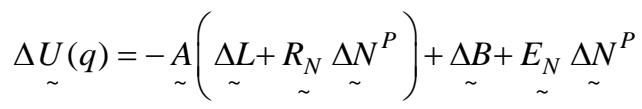

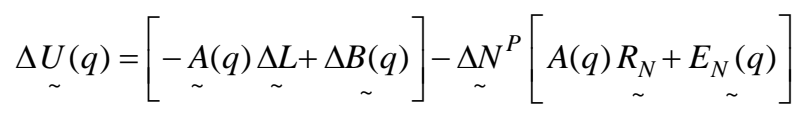

Assim, pode-se escrever a equação (22) da seguinte maneira:

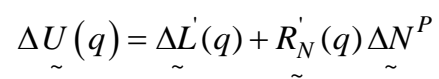

onde

$$
\begin{gathered}
R_{\sim}^{\prime}(q)=-\underset{\sim}{A} \underset{\sim}{R_{N}^{\prime}}+\underset{\sim}{E_{N}} \\
\Delta \underset{\sim}{L^{\prime}}(q)=-\underset{\sim}{A} \underset{\sim}{\Delta L^{\prime}+\Delta B}
\end{gathered}
$$

A equação algébrica das derivadas dos deslocamentos da chapa para qualquer ponto interno é dada pela seguinte equação matricial:

$$
u_{i, j}(q)+\underset{\sim}{H^{\prime}}(q) \Delta \underset{\sim}{U}=\underset{\sim}{G^{\prime}}(q) \underset{\sim}{\Delta P}+\Delta T^{\prime}(q)+\underset{\sim}{E^{\prime}}(q) \underset{\sim}{\Delta N^{P}}
$$

Considerando-se as equações (3), (26), obtém-se a equação matricial relativa à força normal elástica de um ponto interno q. Escrevendo-se essa equação em todos os pontos nodais das células, chega-se à:

$\Delta N^{e(M E C)}=-H^{*} \Delta U+G^{*} \Delta P+\Delta T+E^{N} \Delta N^{P}$

A equação (27) pode ser expressa por:

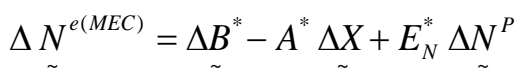

ou ainda:

$$
\Delta \underset{\sim}{N} N^{e(M E C)}=\Delta K_{N}+S_{N} \underset{\sim}{\Delta N^{P}}
$$

onde:

$$
\begin{aligned}
& \underset{\sim}{\Delta K_{N}}=\underset{\sim}{\Delta B_{\sim}^{*}-A_{\sim}^{*} \underset{\sim}{\Delta L}} \\
& S_{\sim}=E_{\sim}^{*}-A_{\sim}^{*} R_{N}
\end{aligned}
$$

Aplicando a lei de Hooke a todos os nós da chapa define-se o vetor de forças normais local $\Delta N^{e}$ como demonstrado em (32).

$$
\Delta N_{\sim}^{e}=\underset{\sim}{C_{N}} \Delta \underset{\sim}{\varepsilon}
$$


onde $\Delta \mathcal{\varepsilon}$ é o vetor de deformações no plano em todos os pontos da chapa;

$$
\left[C_{N}\right]=\left[\begin{array}{ccccc}
{\left[C_{n}\right]_{1}} & {[0]} & . . & . . & {[0]} \\
{[0]} & . . & . . & . . & {[0]} \\
. . & . . & {\left[C_{n}\right]_{i}} & . . & {[0]} \\
. . & . . & . . & . . & {[0]} \\
{[0]} & {[0]} & {[0]} & {[0]} & {\left[C_{n}\right]_{N}}
\end{array}\right]
$$

em que ${\underset{\sim}{N}}_{N}$ é a matriz quadrada, cuja diagonal é composta pelas matrizes elásticas, sendo que os demais termos são nulos.

A fim de deduzir uma equação algébrica do MEC para o esforço normal $N$, que será designado de $N^{M E C}$, considere a equação (3), onde $N$ e $N^{p}$ são forças obtidas localmente, ou seja, para um nó de célula, as forças verdadeiras $N$ são obtidas diretamente a partir das tensões verdadeiras e as forças plásticas $N^{p}$ obtidas subtraindo das forças de tentativa as forças verdadeiras. Analogamente à equação (3), pode-se escrever que:

$$
\Delta N_{\sim}^{M E C}=\Delta N_{\sim}^{e}-\Delta N_{\sim}^{P(M E C)}
$$

Levando-se em conta a expressão das forças normais de tentativa escritas em função das deformações planas (32), tem-se:

$$
\begin{aligned}
& \Delta N_{\sim}^{(\mathrm{MEC})}=C_{N} \Delta \mathcal{\sim}-\Delta N_{\sim}^{P(\mathrm{MEC})} \\
& \text { Onde: } \stackrel{\Delta N_{\sim}^{P(M E C)}}{\sim}=\Delta N_{\sim}^{e(M E C)}-\Delta N
\end{aligned}
$$

Considerando-se a equação (29), tem-se:

$$
\Delta \sim_{\sim}^{P(\mathrm{MEC})}=\Delta{\underset{\sim}{N}}_{N}+{\underset{\sim}{N}}_{N} \Delta{\underset{\sim}{N}}^{P}-\Delta \underset{\sim}{N}
$$

Substituindo-se (36) em (35), obtém-se a equação algébrica para o incremento de forças normais verdadeiras da chapa:

$$
\Delta N^{(\mathrm{MEC})}=C_{N} \Delta \varepsilon-\Delta K_{N}-S_{N} \Delta N^{P}+\Delta N
$$

\section{3- Equação de Resíduos}

Para o problema bidimensional, a equação de resíduos, ou equação de equilíbrio, que deve ser nula em um determinado incremento, é dada por:

$$
R_{N}=\Delta K_{n}-\Delta N_{n}^{M E C}
$$

onde $R_{N}$ é o resíduo- das forças normais; $\Delta K$ o incremento de forças normais elástico; $\Delta N^{M E C}$ é o incremento de forças normais verdadeiro.

O vetor de forças $\Delta K$ é obtido através da formulação linear do MEC para a análise do problema bidimensional, sendo que $\Delta N_{n}^{M E C}$ é calculado levando em consideração a formulação não linear do MEC. Quando a equação (38) for aproximadamente igual à zero tem-se o equilíbrio, obtendo-se os valores reais das incógnitas na placa.

No processo iterativo serão dadas à chapa correções de deformação plana. Então, para que se possa definir o operador tangente consistente, deve-se escrever (38) em termos do incremento de deformação plana $\Delta \varepsilon_{n}$ e das forças normais verdadeiras $\Delta N$ que está relacionado a $\Delta \varepsilon_{n}$ através do tensor elastoplástico $C_{N}^{e p}$.A expressão final para a equação de resíduos é obtida substituindo-se (37) em (38), ou seja:

$$
R_{N}(\Delta \varepsilon)=2 \Delta K_{N}-C_{N} \Delta \varepsilon+S_{N}\left(C_{N} \Delta \varepsilon-\Delta N\right)-\Delta N=0
$$




\section{MATRIZ TANGENTE CONSISTENTE}

A matriz tangente do problema bidimensional é obtida linearizando-se a equação (39) de resíduos, através do Método de Newton-Raphson. Para uma determinada iteração $i$ a correção no estado de deformações planas $\left\{\delta \Delta \varepsilon_{n}^{i}\right\}$ é calculada a partir da seguinte equação:

$$
\left\{R_{N}\right\}_{n}^{i-1}+\frac{\partial\left\{R_{N}\right\}_{n}^{i-1}}{\partial\{\Delta \varepsilon\}_{n}^{i-1}}\{\delta \Delta \varepsilon\}_{n}^{i}=0
$$

ou ainda:

$$
\{\delta \Delta \varepsilon\}_{n}^{i}=\left[-\frac{\partial\left\{R_{N}\right\}_{n}^{i-1}}{\partial\{\Delta \varepsilon\}_{n}^{i-1}}\right]^{-1}\left\{R_{N}\right\}_{n}^{i-1}
$$

Ou seja, a matriz tangente consistente é dada por:

$$
\left[K^{T C}\right]^{i}=-\frac{\partial\left\{R_{N}\right\}_{n}^{i-1}}{\partial\{\Delta \varepsilon\}_{n}^{i-1}}
$$

à:

Derivando-se a equação (39) de resíduos em relação ao incremento de deformação plana, chega-se

$$
\left[K^{T C}\right]_{n}^{i}=\left[S^{\prime}{ }_{N}\right]\left(\left[C_{N}^{e p}\right]_{n}^{i-1}-\left[C_{N}\right]\right)+\left[C_{N}\right]+\left[C_{N}^{e p}\right]_{n}^{i-1}
$$

sendo $\left[C_{N}^{e p}\right]^{i-1}$ uma matriz quadrada, cuja diagonal é composta pelas matrizes elasto-plásticas, e os outros termos são nulos, isto é:

$$
\left[C_{N}^{e p}\right]=\left[\begin{array}{ccccc}
{\left[C_{n}^{e p}\right]_{1}} & {[0]} & . . & . . & {[0]} \\
{[0]} & . . & . . & . . & {[0]} \\
. . & . . & {\left[C_{n}^{e p}\right]_{k}} & . . & {[0]} \\
. . & . . & . . & . . & {[0]} \\
{[0]} & {[0]} & {[0]} & {[0]} & {\left[C_{n}^{e p}\right]_{N}}
\end{array}\right]
$$

sendo $\mathrm{N}$ o número de nós de célula e para um ponto qualquer $\mathrm{k}$ da chapa, $\left[C_{n}^{e p}\right]_{k}$ é dado por:

$$
\left[C_{n}^{e p}\right]_{k(n)}^{i-1}=\frac{\partial N_{n}^{(i-1)}}{\partial \Delta\left(\varepsilon^{2 D}\right)_{n}^{i-1}}=\int_{-h / 2}^{\mathrm{h} / 2} \frac{\partial(\sigma)_{n}^{i-1}}{\partial \Delta(\varepsilon)_{n}^{i-1}} d x_{3}=\int_{-h / 2}^{\mathrm{h} / 2}\left[C^{e p}\right]_{k(n)}^{i-1} d x_{3}=h C^{e p}
$$

onde $C^{e p}$ que é obtido a partir do modelo constitutivo adotado, é a matriz constitutiva que relaciona a tensão real com a deformação total.

\section{PROCESSO INCREMENTAL ITERATIVO}

O algoritmo que rege o processo para a análise não linear de chapas é o seguinte: para uma iteração $i$ de um incremento $n$, segue-se os seguintes passos:

1) Calculam-se os incrementos de forças normais elásticas $\Delta K_{n}$, referentes a todos os pontos nodais das células.

2) Quando $i=0$ é feito o cálculo dos incrementos de deformações totais $\{\Delta \varepsilon\}_{n}^{i}$ através da lei de Hooke (32).Caso a equação de resíduo (39) não for satisfeita, houve a plastificação de algum ponto, de- 
vendo-se fazer o processo iterativo $(i \geq 1)$ descrito no passo 3 para alcançar o equilíbrio da chapa.

3) Se $i \geq 1$ : atualiza-se a matriz tangente global $\left[K_{(\eta)}^{T C(i)}\right]$ (43) e calculam-se as correções que devem ser dadas ao incremento de deformações planas $\left.\{\delta \Delta \varepsilon\}_{n}\right\}^{2}(41)$, obtendo-se o novo estado de deformações na chapa:

$$
\{\Delta \varepsilon\}_{n}^{i+1}=\{\Delta \varepsilon\}_{n}^{i}+\{\delta \Delta \varepsilon\}_{n}^{i}
$$

4) Para cada ponto nodal das células, procede-se da seguinte maneira:

4.1) Verifica-se o modelo constitutivo para o ponto de Gauss ig definido ao longo da espessura da chapa, seguindo os seguintes passos:

4.1.1) Obtém-se o incremento de tensões elásticas, ou de tentativa, $\left\{\Delta \sigma^{e}\right\}_{n}^{i+1}$ através da equação:

$$
\left\{\Delta \sigma^{e}\right\}_{n}^{i+1}=[C]\{\Delta(\varepsilon)\}_{n}^{i+1}
$$

sendo $[C]$ o tensor elástico dado pela lei de Hooke

4.1.2) Soma-se (47) ao estado de tensão verdadeiro do incremento anterior convergido, obtendo-se as tensões totais $\left\{\sigma^{e}\right\}^{i+1}$, ou seja:

$$
\left\{\sigma^{e}\right\}_{n}^{i+1}=\{\sigma\}_{n-1}+\left\{\Delta \sigma^{e}\right\}_{n}^{i+1}
$$

4.1.3) Com as tensões totais verifica-se o modelo constitutivo, obtendo-se o vetor de tensão verdadeiro $\{\sigma\}_{n}^{i+1}$ e o incremento de tensão verdadeiro $\{\Delta \sigma\}_{n}^{i+1}=\{\sigma\}_{n}^{i+1}-\{\sigma\}_{n-1}$ para o ponto em questão. Se a iteração não for elástica, deve-se atualizar também a relação constitutiva elasto-plástica $\left[C^{e p}\right]$ definida em (45).

4.2) Calcula-se o vetor dos incrementos de forças normais verdadeiras $\Delta N^{(i+1)}$ e o vetor dos incrementos de forças normais plásticas $\Delta N_{n}^{p(i+1)}$ do ponto $k$, além da matriz quadrada $\left[C_{N}^{e p}\right]$ composta das matrizes elasto-plásticas (44) :

$$
\begin{aligned}
& \left\{\Delta N^{(i+1)}\right\}^{k}=h \Delta \sigma_{n}^{i+1} \\
& \left\{\Delta N_{n}^{p(i+1)}\right\}=\left[C_{N}\right]\{\Delta \varepsilon\}-\left\{\Delta N^{i+1}\right\}
\end{aligned}
$$

5) Segue-se o mesmo procedimento para todos os pontos nodais das células. Através da equação (39) calculam-se os resíduos de forças normais $R_{N}^{(i+1)}$.Se os resíduos não forem nulos, de acordo com a tolerância adotada, passa-se à próxima iteração, voltando ao passo 3. Caso o critério de convergência seja verificado, passa-se ao incremento $(n+1)$ seguinte ao passo 1 com $i=0$.

Ao final de um incremento de carga, os incrementos dos deslocamentos e forças de superfície que são incógnitos no contorno da chapa são dados por:

$$
\{\Delta X\}_{n}=\{\Delta L\}_{n}+\left[R_{N}\right]\left\{\Delta N^{p}\right\}_{n}
$$
onde $\Delta L$ representa a resposta elástico linear e $\left\{\Delta N^{p}\right\}_{n}$ contém os vetores $\left\{\Delta N^{p}\right\}^{k}$ de todos os pontos
da chapã.

O incremento de força normal real na chapa é dado pela equação (37).

\section{EXEMPLOS NUMÉRICOS}

Neste artigo estudaram-se três exemplos. No primeiro apresenta-se uma chapa de material genérico carregada com forças normais e tangenciais. No segundo e no terceiro exemplo utilizou-se uma chapa de alumínio, sendo uma com furo quadrado e outra com furo circular e ambas submetidas a carregamentos normais. Nos exemplos foi analisada a convergência dos resultados pela adição de elementos no contorno e células no domínio, além de mostrar a convergência quadrática do erro ao longo do processo iterativo. Para todos os exemplos numéricos adotou-se o modelo elasto-plástico de Von Mises para modelar o 
comportamento do material.

Note que em todos os exemplos apresentados são definidos nós duplos nos cantos. Assim, o nó anterior ao canto é definido como nó da célula anterior e o nó posterior ao canto como nó da célula posterior. Portanto, nas figuras que mostram a discretização da placa (veja figuras 6, 10 e 15) os lados das células, cujas extremidades correspondem ao nó de canto, foram representadas não coincidentes apenas para definir qual nó estava sendo considerado para cada célula. No entanto, na integração numérica das células esses lados são considerados coincidentes.

\subsection{Chapa retangular}

Na figura 5 tem-se uma chapa de comprimento $200 \mathrm{~cm}$, largura $50 \mathrm{~cm}$ e espessura de $20 \mathrm{~cm}$. A mesma está submetida a uma força de $1,0 \mathrm{kN} / \mathrm{cm}^{2}$, sendo que, multiplicando pela espessura tem-se uma carga de $20 \mathrm{kN} / \mathrm{cm}$. Para as propriedades do material adotaram-se: módulo de elasticidade $E=1000 \mathrm{kN} / \mathrm{cm}^{2}$, módulo plástico $E^{e p}=100 \mathrm{kN} / \mathrm{cm}^{2}$, Poisson $v=0,3$, tensão de escoamento de $\sigma_{y}=1 \mathrm{kN} / \mathrm{cm}^{2}$ e coeficiente de encruamento $K=111,11 \mathrm{kN} / \mathrm{cm}^{2}$.

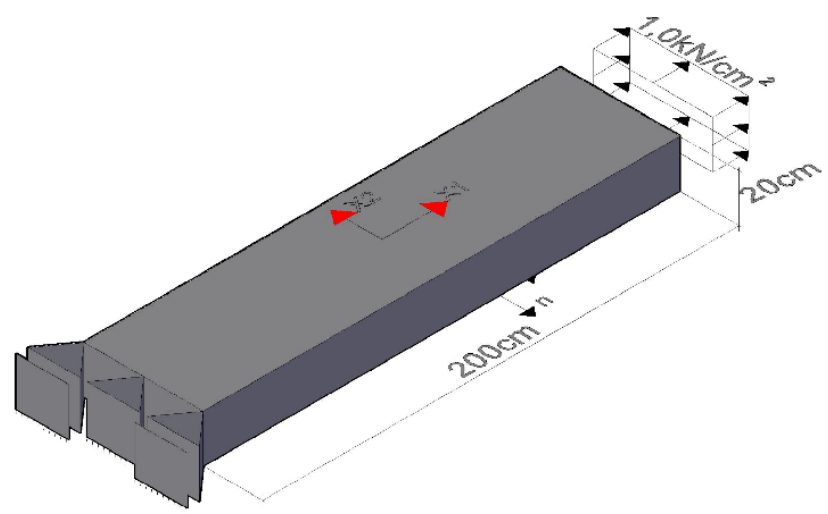

Figura 5: Exemplo de uma chapa com carregamento normal

As vinculações e condições de contorno estão apresentadas na figura 5, onde ao longo de um dos lados menores restringe-se o deslocamento na direção $X_{1}$ para todos os nós e na direção $X_{2}$ apenas para o ponto médio. No lado oposto a esse, aplica-se força na direção normal ao contorno de valor $p_{n}=20 \mathrm{kN} /$ $\mathrm{cm}$ e os dois lados maiores são adotados livres $\left(p_{s}=p_{n}=0\right)$. A força prescrita é aplicada ao longo de oito incrementos, onde se adota $\Delta \beta=1$ para o primeiro incremento de carga, $\Delta \beta=0,5$ para o segundo e $\Delta \beta=0,25$ para os outros, sendo $\beta$ o fator de carga acumulado e $\Delta \beta$ o incremento do fator de carga. Note que a solução analítica desse exemplo é conhecida, sendo o deslocamento em um ponto $p$ qualquer da chapa dado por $u_{n}^{p}=u_{n-1}^{p}+\Delta \varepsilon x_{1}^{p}$ Portanto, obtêm-se os seguintes deslocamentos para o ponto 9 (ver figura 6) correspondentes aos fatores de carga $\beta=1, \beta=2$ e $\beta=3$ :

$$
\begin{aligned}
& u_{\beta=1}=\varepsilon l=0,001 \times 200=0,2 \mathrm{~cm} \\
& u_{\beta=2}=\varepsilon l=0,011 \times 200=2,2 \mathrm{~cm} \\
& u_{\beta=3}=\varepsilon l=0,021 \times 200=4,2 \mathrm{~cm}
\end{aligned}
$$

Na análise com o MEC foram utilizadas duas malhas; a primeira com 4 elementos no contorno e 8 células e a segunda com 8 elementos no contorno e 24 células, que está representada na figura 6, onde os pontos vermelhos indicam os nós dos elementos. Observe que está indicado em linha vermelha o primeiro elemento de cada aresta da chapa. 


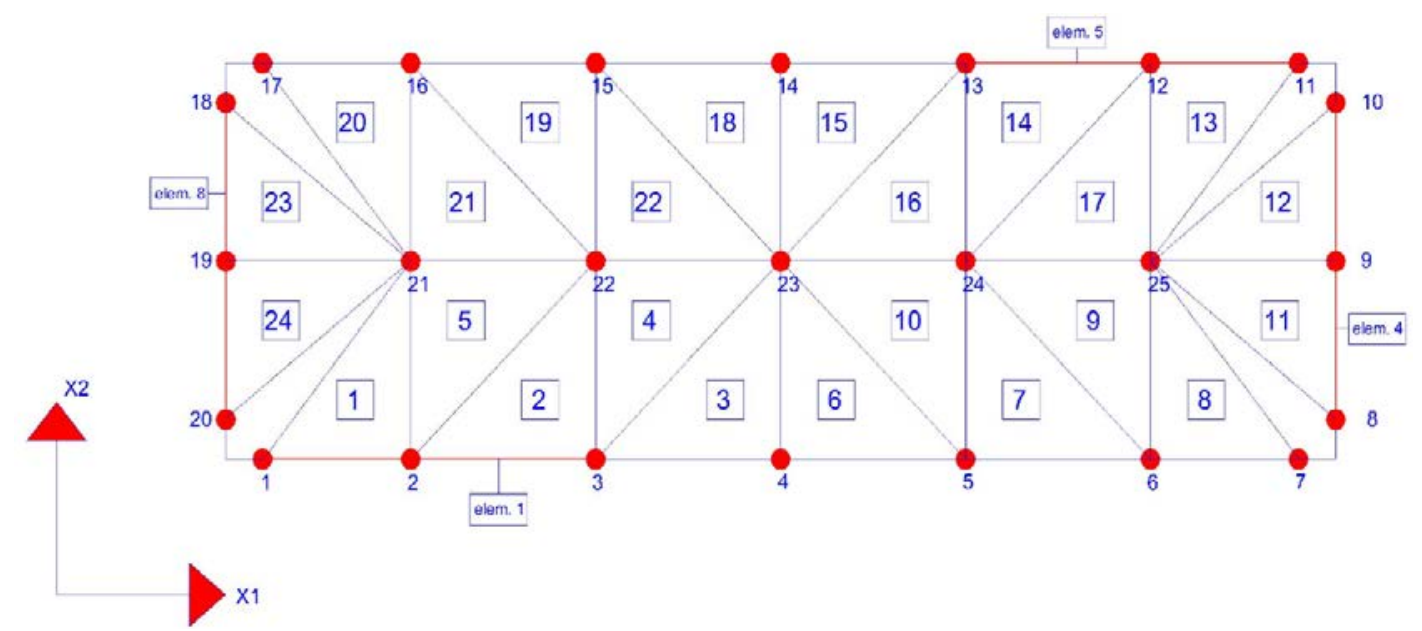

Figura 6: Malha 24(8) com vinte e quatro células no domínio e oito elementos no contorno

Na figura 7 tem-se o deslocamento do ponto 9 na direção de $X_{1}$, sendo que na legenda do gráfico o valor fora do parêntese corresponde a quantidade de células no domínio, e o valor dentro do parêntese a quantidade elementos no contorno.

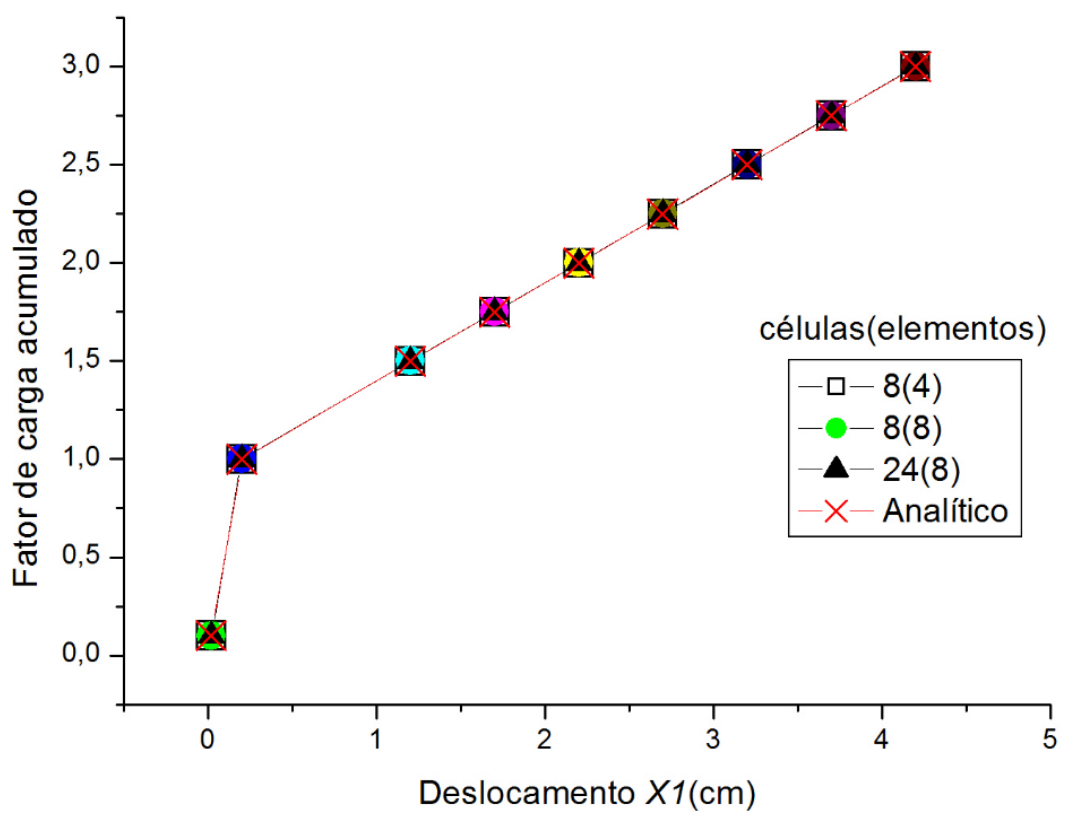

Figura 7: Deslocamento do ponto 9 para análise não linear com carregamento normal

Na tabela 1 está demonstrada a convergência quadrática do resíduo (erro) ao longo do processo iterativo para dois incrementos de carga: $\beta=1,5$ e $\beta=3$. Nessa tabela foi considerada a malha 8(8), ou seja, com oito elementos no contorno e oito células no domínio. Note que o resíduo de forças normais é calculado pela equação (39). 
Tabela 1: Convergência quadrática do resíduo para a malha 8(8)

\begin{tabular}{c|c|c}
\hline & INCREMENTO 3 & INCREMENTO 8 \\
\hline & $\Delta \beta=0,5$ & $\Delta \beta=0,25$ \\
\hline & $\beta=1,5$ & $\beta=3$ \\
\hline ITERAÇÃO & \multicolumn{2}{|c}{ RESÍDUO } \\
\hline 1 & $9,78 \mathrm{E}-01$ & $9,78 \mathrm{E}-01$ \\
\hline 2 & $8,00 \mathrm{E}-01$ & $8,09 \mathrm{E}-01$ \\
\hline 3 & $1,89 \mathrm{E}-02$ & $7,57 \mathrm{E}-03$ \\
\hline 4 & $7,61 \mathrm{E}-05$ & $2,93 \mathrm{E}-05$ \\
\hline 5 & $4,89 \mathrm{E}-11$ & $1,06 \mathrm{E}-11$ \\
\hline
\end{tabular}

No gráfico da figura 8 estão os valores da tabela 1, onde se percebe que o resíduo diminui significativamente entre uma iteração e outra, sendo observada a convergência quadrática do erro.

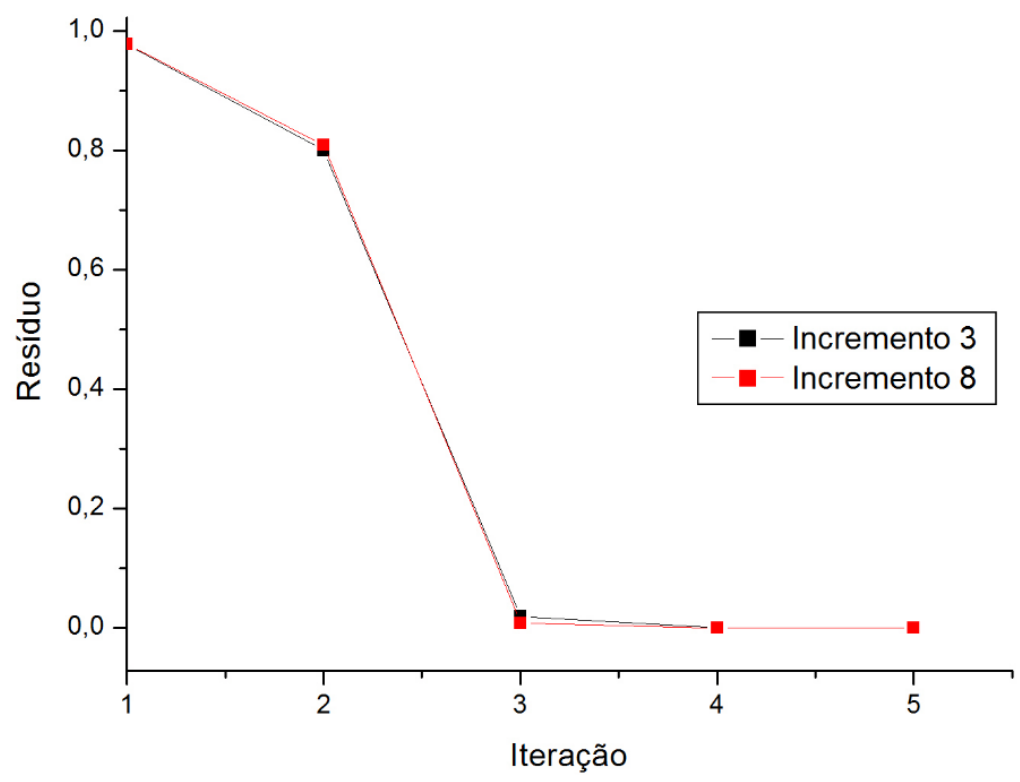

Figura 8: Resíduo nos incrementos da malha 8(8)

Na tabela 2 está demonstrada a convergência quadrática do resíduo ao longo do processo iterativopara a malha de vinte e quatro células no domínio e oito elementos no contorno, referentes também ao terceiro e oitavo incrementos de carga.

Tabela 2: Convergência da malha 24(8)

\begin{tabular}{c|c|c}
\hline & INCREMENTO 3 & INCREMENTO 8 \\
\hline & $\Delta \beta=0,5$ & $\Delta \beta=0,25$ \\
\hline & $\beta=1,5$ & $\beta=3$ \\
\hline ITERAÇÃO & \multicolumn{2}{|c}{ RESíDUO } \\
\hline 1 & $9,78 \mathrm{E}-01$ & $9,78 \mathrm{E}-01$ \\
\hline 2 & $8,09 \mathrm{E}-01$ & $8,20 \mathrm{E}-01$ \\
\hline 3 & $7,57 \mathrm{E}-03$ & $3,91 \mathrm{E}-04$ \\
\hline 4 & $2,93 \mathrm{E}-05$ & $1,19 \mathrm{E}-07$ \\
\hline 5 & $1,06 \mathrm{E}-11$ & $2,08 \mathrm{E}-15$ \\
\hline
\end{tabular}


Seja agora a mesma placa descrita na figura 5, mudando-se apenas as forças aplicadas. Além da força normal, aplica-se também uma força tangencial como mostrado na figura 9.

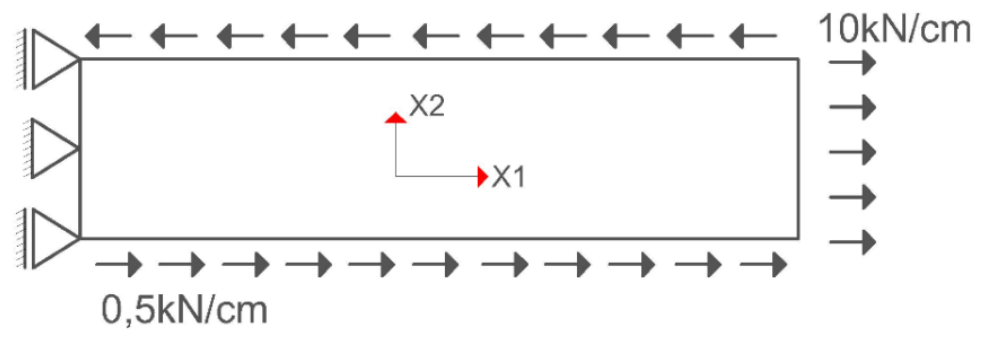

Figura 9: Chapa com carregamento normal $\left(p_{n}\right)$ e tangencial $\left(p_{s}\right)$

Para este exemplo tem-se a discretização do domínio em 24,48 e 192 células e para o contorno foram considerados 26 e 52 elementos. Na figura 10 tem-se a malha 192(52), onde os pontos em vermelho indicam os nós de células definidas no domínio, sendo algum desses coincidentes com os nós sobre o contorno, que também são definidos nos elementos de contorno.

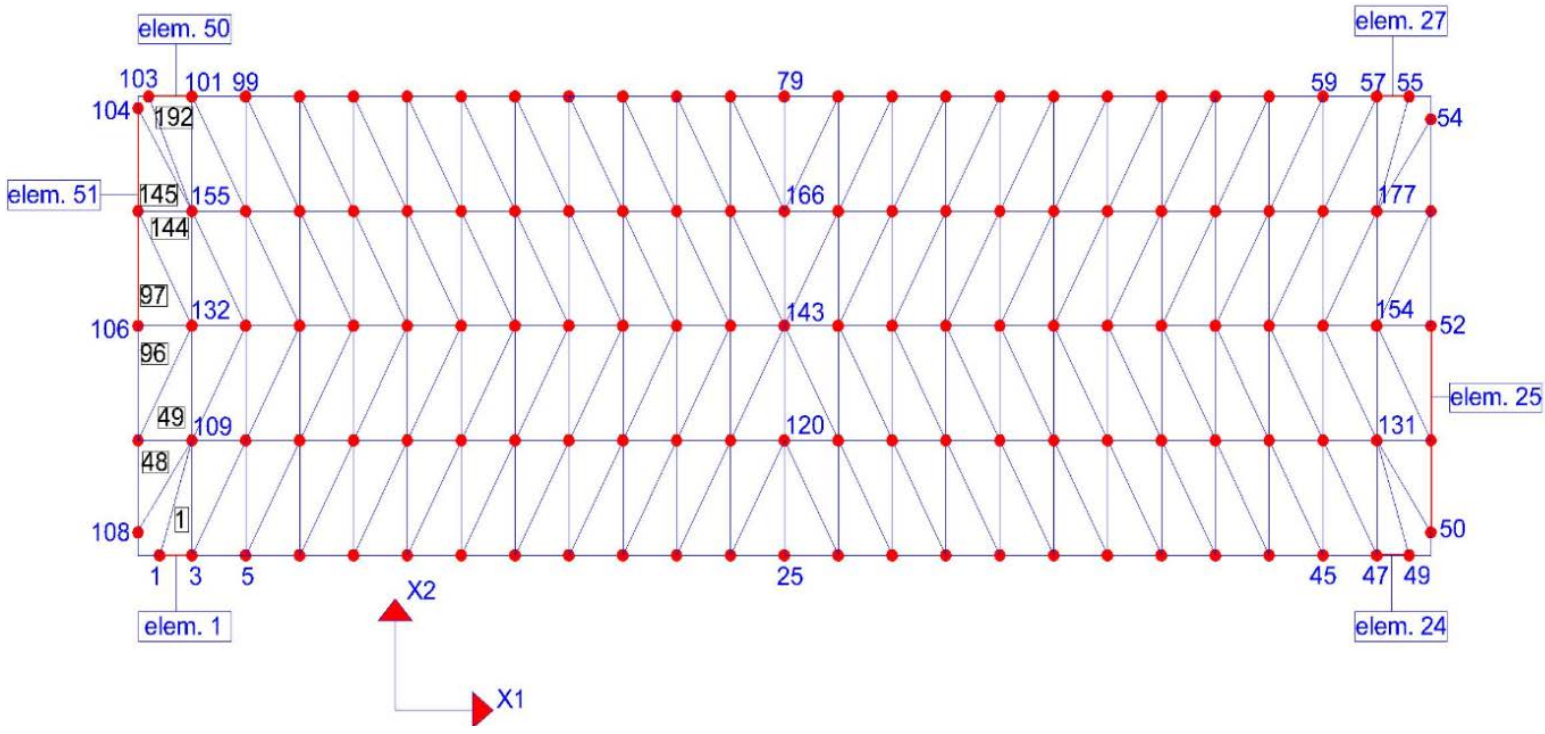

Figura 10: Malha 192(52) com cinquenta e dois elementos no contorno e cento e noventa e duas células no domínio

Nas figuras 11 e 12, mostram-se os deslocamentos para o ponto 52 (ver figura 10) ao longo do processo incremental de carga. 


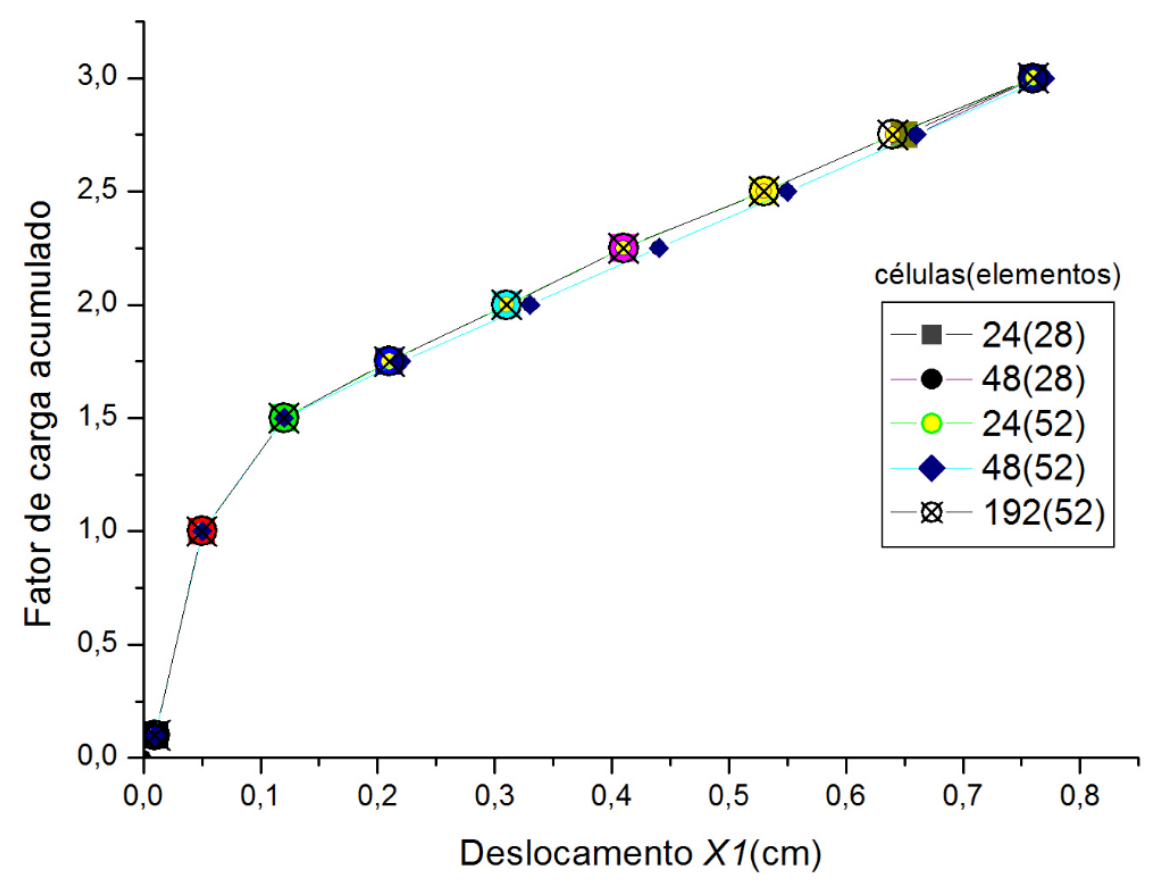

Figura 11: Deslocamento $X 1$ do ponto 52

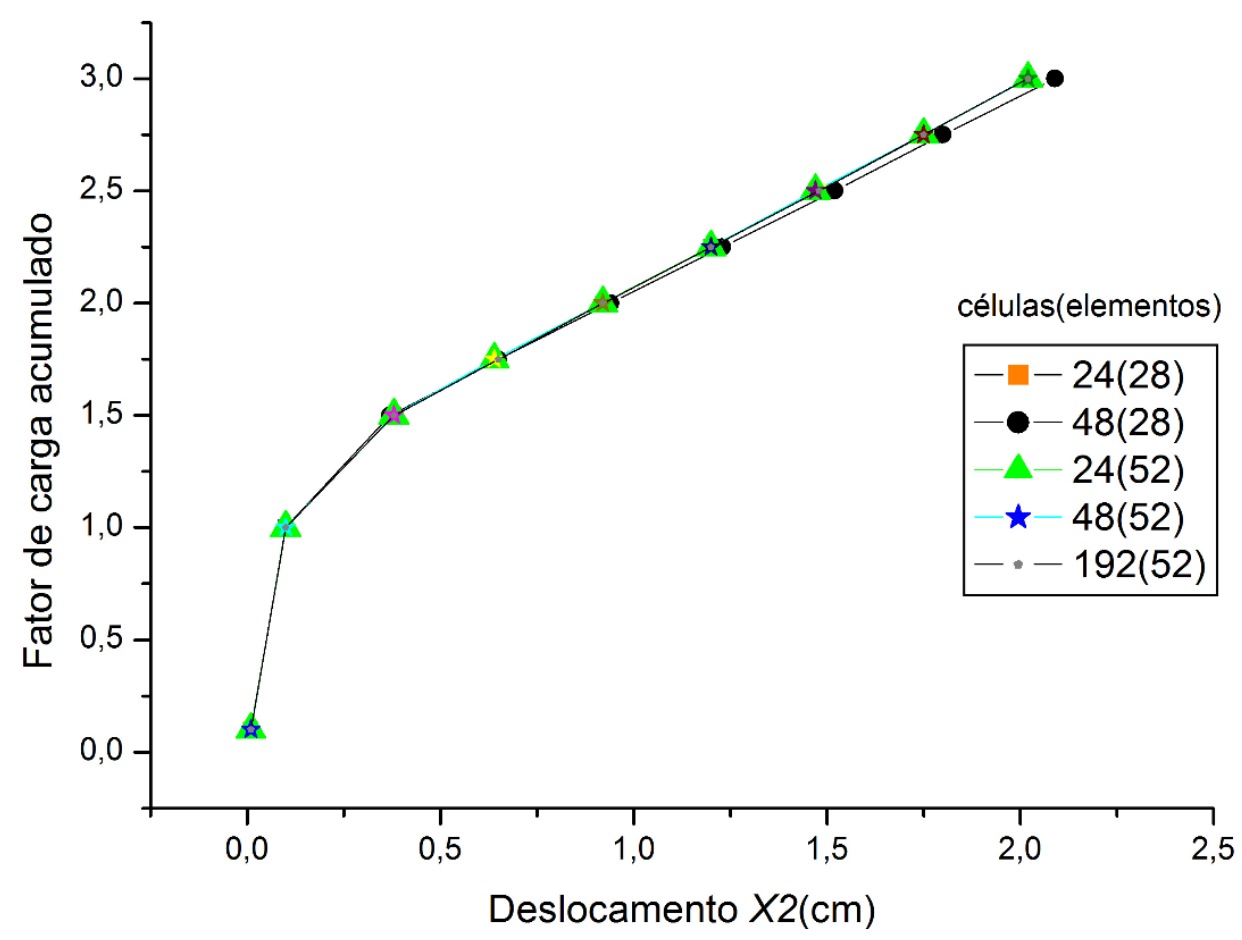

Figura 12: Deslocamento $X 2$ do ponto 52

Note que ambas as malhas de quarenta e oito células apresentam bons resultados. Na tabela 3 está demonstrada a convergência quadrática do resíduo ao longo dos processos iterativos do terceiro e oitavo incrementos de carga, para a malha 48(28). 
Tabela 3: Convergência quadrática do resíduo para a malha 48(28)

\begin{tabular}{c|c|c}
\hline & INCREMENTO 3 & INCREMENTO 9 \\
\hline & $\Delta \beta=0,5$ & $\Delta \beta=0,25$ \\
\hline & $\beta=1,5$ & $\beta=3$ \\
\hline ITERAÇÃO & \multicolumn{2}{|c}{ RESíDUO } \\
\hline 1 & $9,51 \mathrm{E}-01$ & $9,51 \mathrm{E}-01$ \\
\hline 2 & $4,76 \mathrm{E}-01$ & $7,82 \mathrm{E}-01$ \\
\hline 3 & $5,23 \mathrm{E}-03$ & $8,93 \mathrm{E}-04$ \\
\hline 4 & $2,24 \mathrm{E}-06$ & $9,47 \mathrm{E}-08$ \\
\hline 5 & $2,51 \mathrm{E}-11$ & \\
\hline
\end{tabular}

Na tabela 4 a convergência quadrática do resíduo é verificada para a malha 192(52).

Tabela 4: Convergência quadrática do resíduo para a malha 192(52)

\begin{tabular}{c|c|c}
\hline & INCREMENTO 3 & INCREMENTO 9 \\
\hline & $\Delta \beta=0,5$ & $\Delta \beta=0,25$ \\
\hline & $\beta=1,5$ & $\beta=3$ \\
\hline ITERAÇÃo & \multicolumn{2}{|c}{ RESíDUO } \\
\hline 1 & $9,85 \mathrm{E}-01$ & $9,85 \mathrm{E}-01$ \\
\hline 2 & $5,41 \mathrm{E}-01$ & $8,11 \mathrm{E}-01$ \\
\hline 3 & $5,35 \mathrm{E}-03$ & $1,35 \mathrm{E}-04$ \\
\hline 4 & $2,31 \mathrm{E}-06$ & $1,59 \mathrm{E}-08$ \\
\hline 5 & $2,63 \mathrm{E}-11$ & \\
\hline
\end{tabular}

\section{2: Chapas de alumínio com furos circular e quadrado}

Para este exemplo têm-se as duas chapas de alumínio como visto na figura 13. As características do material são: módulo de elasticidade de $E=70 \mathrm{GPa}$, tensão de escoamento de $\sigma_{y}=243 \mathrm{Mpa}$; módulo de encruamento $K=0,032 x E$; coeficiente de Poisson $v=0,2$.

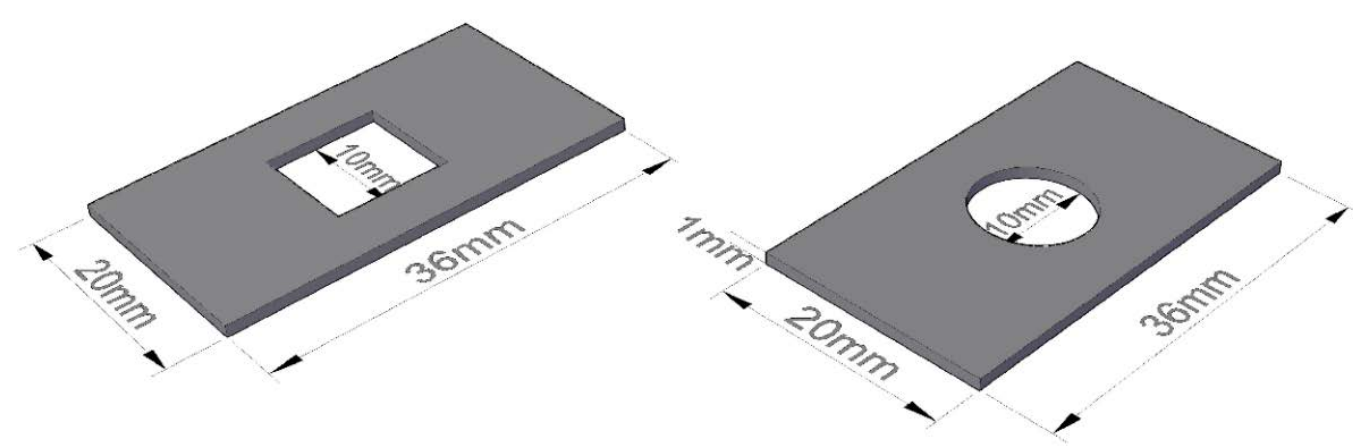

Figura 13: Chapas de alumínio com furos quadrado e circular

As vinculações e condições de contorno estão apresentadas na figura 14, onde uma carga de 121,5 $\mathrm{N} / \mathrm{mm}$ é aplicada ao longo dos lados menores. Note que devido à simetria do problema, será analisado apenas $1 / 4$ da placa (parte hachurada da figura 14). 

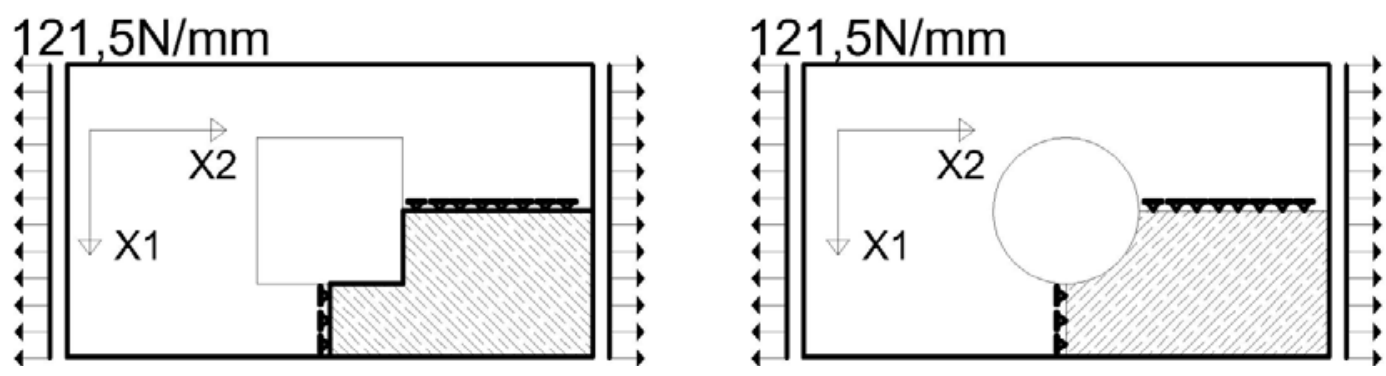

Figura 14: Chapas de alumínio com carregamentos normais

Considere inicialmente a placa com furo quadrado, onde se utilizou uma malha de duzentos e seis pontos no contorno para definição dos elementos de contorno. Para a discretização do domínio foram consideradas três diferentes malhas, contendo 24,88 e 250 células, respectivamente, sendo esta última vista na figura 15. Como condição de contorno em deslocamentos, restringiu-se o deslocamento na direção de X2 para os pontos 166 até 206 (ver figura 15) e o deslocamento na direção de X1 para os pontos 43 até 63 (ver figura 15). A fim de verificar a formulação, os deslocamentos e as forças foram comparadas com o software de elementos finitos Ansys.

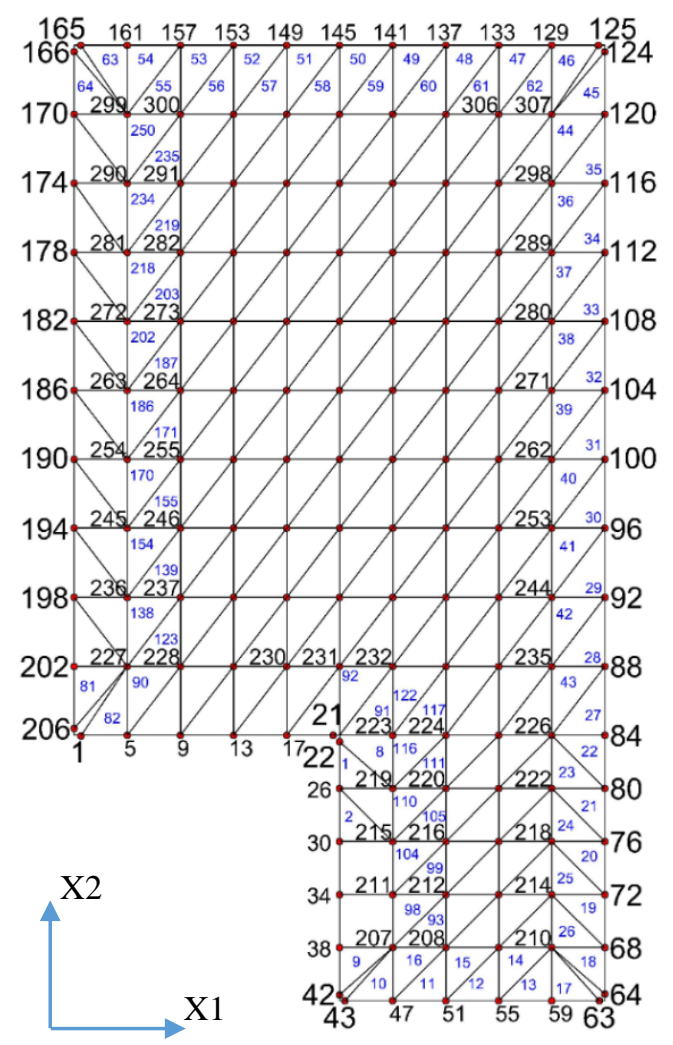

Figura 15: Malha 250(100) com duzentos e cinquenta células no domínio e cem elementos no contorno

Na figura 16 tem-se o deslocamento do ponto 1 ao longo do processo incremental, onde para a análise no Ansys utilizou-se o elemento solid 185. 


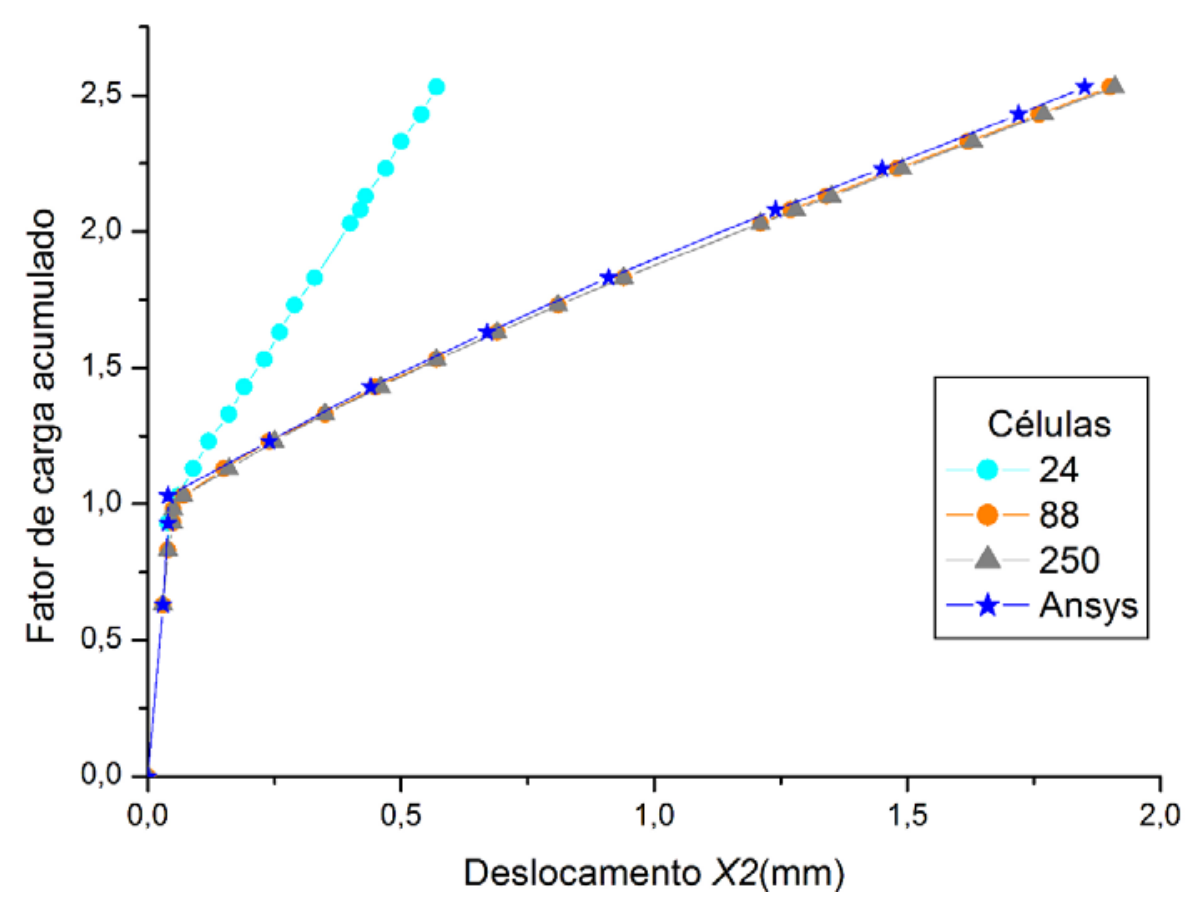

Figura 16: Deslocamento do ponto 1 para malhas com 24, 88 e 250 células e no Ansys

Percebe-se que a malha de vinte e quatro células apresenta resultados discrepantes das demais, ao passo que os deslocamentos obtidos com as malhas de 88 e 250 células são semelhantes aos valores calculados com o Ansys. Observa-se que para fator de carga $\beta=0,83$ houve o início de plastificação do material, porém a plastificação se torna mais acentuada a partir de $\beta=1$, ou seja, para carga de $p_{n}=121,5 \mathrm{~N} /$. A convergência quadrática do resíduo ao longo do processo iterativo para os incrementos com $\beta=0,83$ e $\beta=2,53$ são demonstrados na tabela 5, para a malha 250(100).

Tabela 5: Convergência quadrática do resíduo para a malha 250(100) com furo quadrado

\begin{tabular}{c|c|c}
\hline & INCREMENTO 2 & INCREMENTO 20 \\
\hline & $\Delta \beta=0,2$ & $\Delta \beta=0,05$ \\
\hline & $\beta=0,83$ & $\beta=2,53$ \\
\hline ITERAÇÃO & \multicolumn{2}{|c}{ RESÍDUO } \\
\hline 1 & $9,80 \mathrm{E}-01$ & $9,80 \mathrm{E}-01$ \\
\hline 2 & $1,12 \mathrm{E}-01$ & $9,24 \mathrm{E}-01$ \\
\hline 3 & $1,96 \mathrm{E}-02$ & $1,19 \mathrm{E}-04$ \\
\hline 4 & $3,26 \mathrm{E}-03$ & $6,56 \mathrm{E}-07$ \\
\hline 5 & $1,65 \mathrm{E}-06$ & $4,49 \mathrm{E}-12$ \\
\hline
\end{tabular}

Na figura (17) têm-se as forças obtidas para os pontos 43 até 63 no último incremento de carga. Note que os resultados obtidos com a malha com 24 células não foram apresentados por serem muito discrepantes dos demais. 


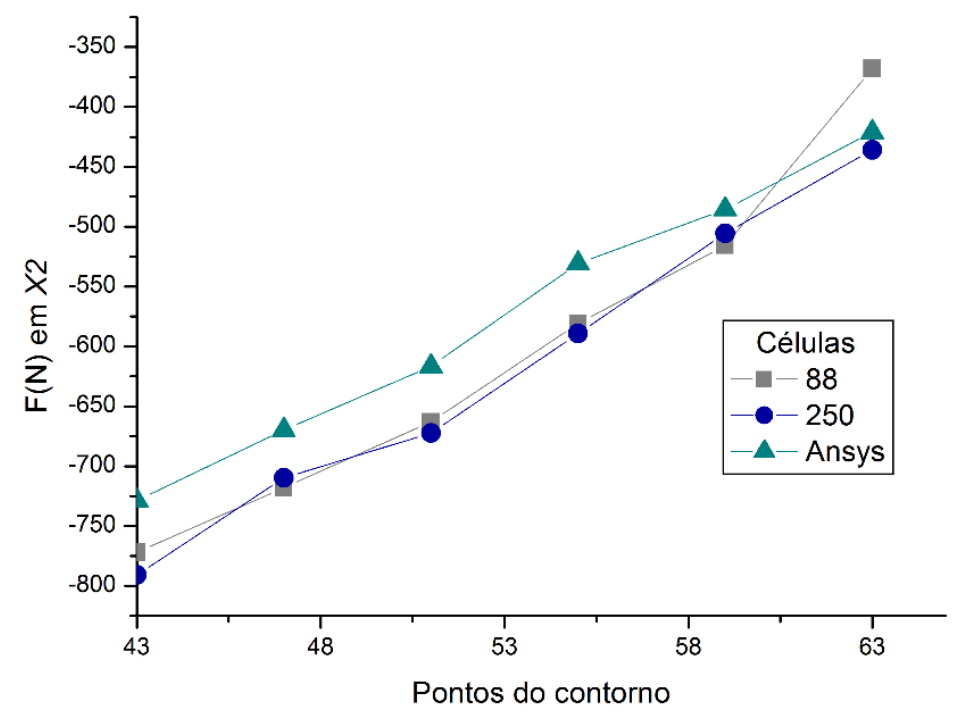

Figura 17: Forças dos pontos quarenta e três a sessenta e três da chapa com furo quadrado

Note que os resultados obtidos com as duas malhas do MEC foram muito próximos, mostrando a convergência dos resultados, sendo esses similares àqueles obtidos com o Ansys.

Seja agora a placa com furo circular, onde se adotam 186 nós para a discretização do contorno em elementos. As vinculações e condições de contorno são idênticas as apresentadas na figura 14 com carga de $121.5 \mathrm{~N} / \mathrm{mm}$ na direção de $p_{n}$. Para o domínio foram consideradas quatro discretizações: 49, 88, $276 \mathrm{e}$ 450 células, sendo esta última vista na figura 18. Esse exemplo também foi analisado com o Ansys, a fim de comparar os resultados numéricos.

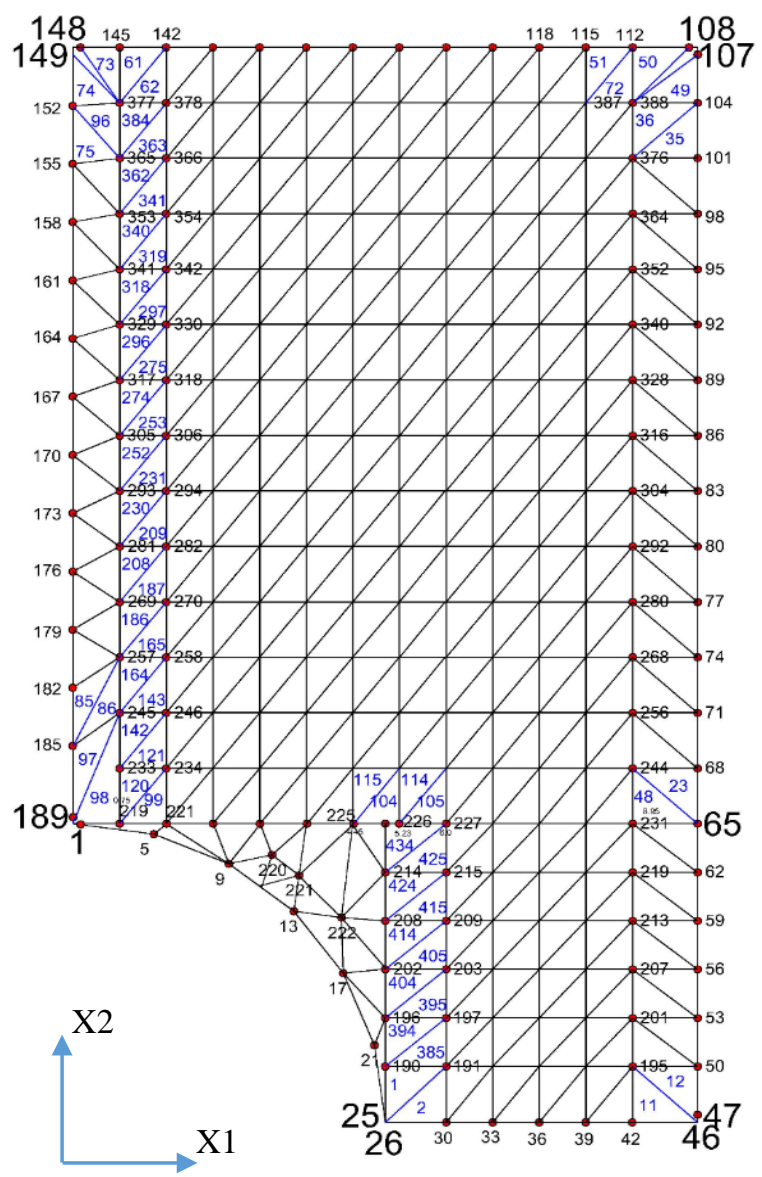

Figura 18: Malha 450(92) com quatrocentos e cinquenta células no domínio e noventa e dois elementos no contorno 
O deslocamento do ponto 1 ao longo do processo incremental de carga está mostrado na figura 19 para as diversas malhas analisadas com o MEC e para o Ansys, onde se utilizou utilizado o elemento solid 185.

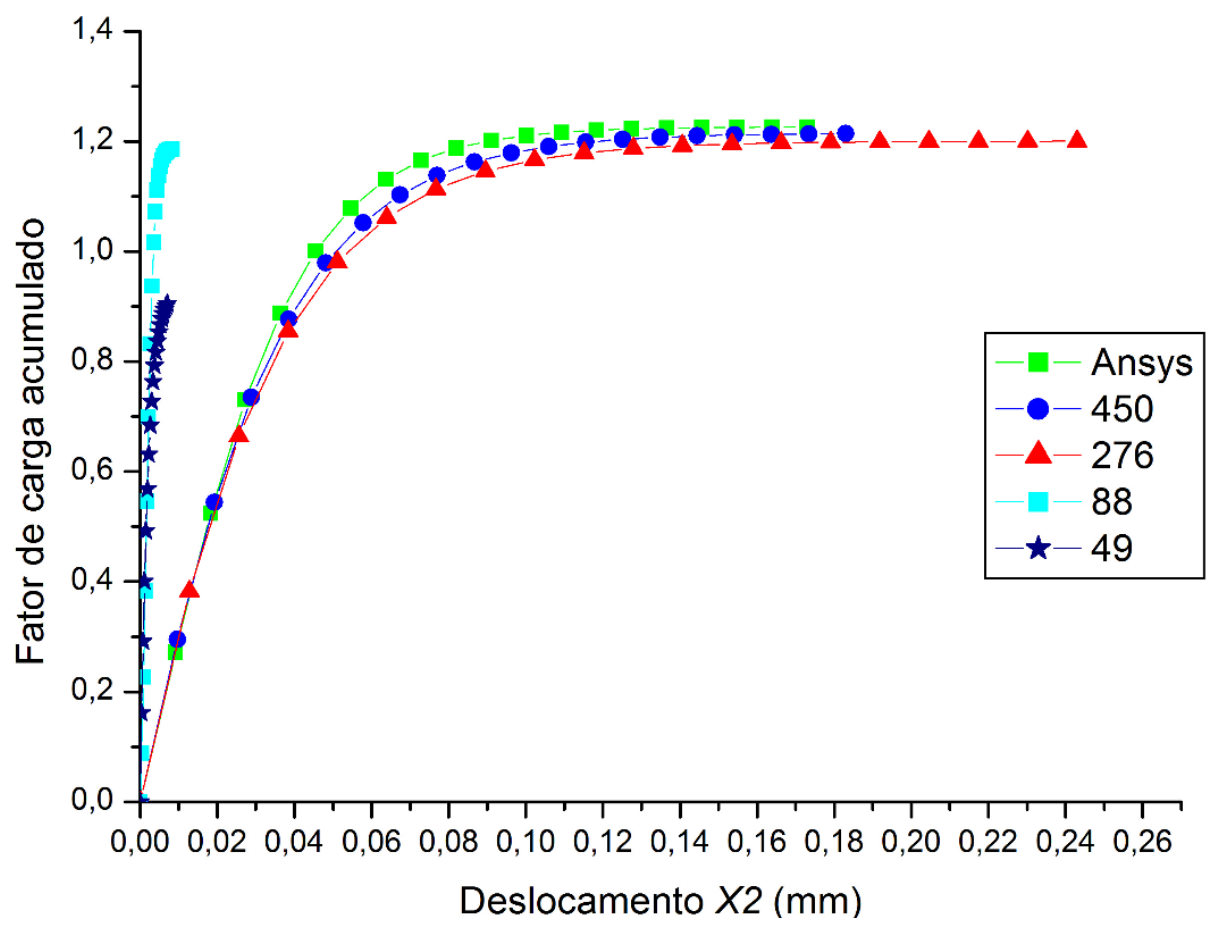

Figura 19: Deslocamento do ponto 1 para malhas 49,276 e 460 e no Ansys

Pode-se observar que, para o furo circular necessita-se de uma maior discretização do domínio para a obtenção da convergência dos resultados numéricos com o MEC. Note também que os deslocamentos obtidos com a malha mais refinada (450 células) foram semelhantes àqueles obtidos com o Ansys. Na tabela 6 mostra-se a convergência quadrática do resíduo para a malha 450(92) ao longo do processo iterativo dos incrementos 2 e 14 .

Tabela 6: Convergência quadrática do resíduo para a malha 450(92) com furo circular

\begin{tabular}{c|c|c}
\hline & INCREMENTO 2 & INCREMENTO 14 \\
\hline & $\Delta \beta=0,2$ & $\Delta \beta=0,02$ \\
\hline ITERAÇÃO & $\beta=0,43$ & $\beta=1,25$ \\
\hline 1 & $9,86 \mathrm{E}-01$ & $9,86 \mathrm{E}-01$ \\
\hline 2 & $5,01 \mathrm{E}-01$ & $9,90 \mathrm{E}-01$ \\
\hline 3 & $1,43 \mathrm{E}-01$ & $5,11 \mathrm{E}-03$ \\
\hline 4 & $1,35 \mathrm{E}-02$ & $8,06 \mathrm{E}-04$ \\
\hline 5 & $2,78 \mathrm{E}-05$ & $1,36 \mathrm{E}-07$ \\
\hline 6 & $1,01 \mathrm{E}-10$ & $1,59 \mathrm{E}-12$ \\
\hline
\end{tabular}

Percebe-se que o erro do processo iterativo diminui rapidamente, com taxa quadrática, mesmo para esse exemplo que possui maior complexidade que os anteriores. As forças no último incremento e referentes aos nós 26 até 46 estão plotados na figura 20. Note que o ponto de colocação para essas forças é adotado no interior da célula, a 0,163 milímetros de distância do centro da chapa. Na figura 20 pode-se observar a convergência dos resultados numéricos para as duas malhas do MEC, além de resultados semelhantes àqueles obtidos com o Ansys. 


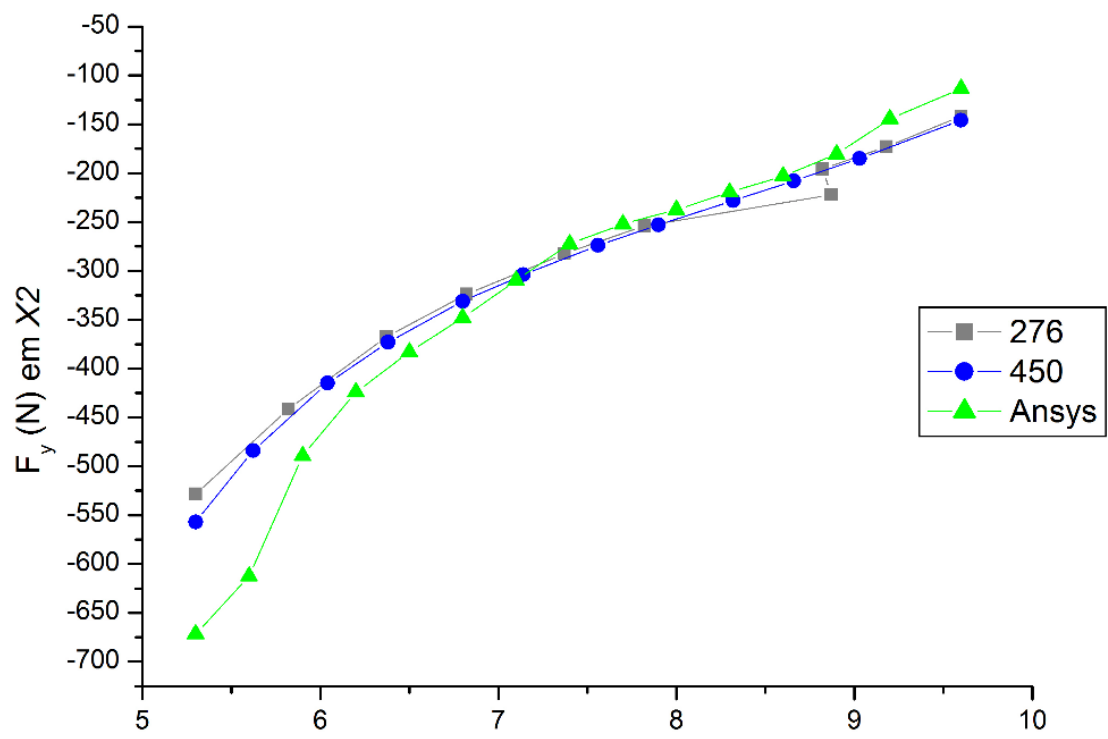

Figura 20: Forças nos pontos de momentos da chapa circular

\section{CONCLUSÕES}

O presente trabalho aplicou a teoria do método dos elementos de contorno para análise de chapas com não linearidade física. Note que nas análises não lineares necessita-se além da discretização do contorno em elementos, a discretização do domínio em células. Na formulação do MEC apresentada, considera-se o operador tangente consistente no cálculo da correção de deformações a ser aplicada ao longo do processo iterativo, o que leva a uma taxa de convergência quadrática do resíduo, mesmo para exemplos mais complexos. Percebeu-se que utilizando uma malha que apresentava bons resultados na análise linear, necessitou-se de pouca adição de células no domínio para se obter a convergência dos resultados. Além disso, a fim de verificar a formulação, os resultados numéricos foram comparados com aqueles obtidos com o Ansys, tendo sido bem similares.

Portanto, tem-se que a utilização do método dos elementos de contorno apresentou-se eficiente nas análises com não linearidade física, obtendo bons resultados mesmo para malhas pouco refinadas.

\section{BIBLIOGRAFIA}

[1] BREBBIA, C.A, TELLES, J.C.F, WROBEL, L.C. Boundary element techniques. Theory and applications in engineering. Springer, Berlin/New York, 1984.

[2] BONNET, M. Équations Intégrales et éléments de fronteire: applications em mécanique des solides et des fluides. Paris, CNRSÉditions/Eyrolles, 1995.

[3]BONNET, M, MUKHERJE, E. "SImplicit BEM formulations for usual and sensivity problems in elasto-plasticity using the consistente tangent operator concept”, Int J Solids Struct, v.33,n.30, pp.4461-4480, 1996.

[4] SOUZA NETO, E. A, PERIC, D., OWEN, D.R.J. Computational methods for plasticity: theory and applications, Wiley, Chichester, 2008.

[5] CHUEIRI, L.H.M. Formulação do método dos elementos de contorno para análise elastoplástica de chapas, Tese de D.Sc., Escola de Engenharia de São Carlos, Universidade de São Paulo, 1994.

[6] FERNANDES, G.R. O método dos elementos de contorno aplicado à análise não linear de chapas. Dissertação M.Sc., Escola de Engenharia de São Carlos, Universidade de São Paulo, 1998.

[7] FERNANDES, G.R. Analise não linear de estruturas de pavimentos de edifícios através do método dos elementos de contorno,Tese de D.Sc., Escola de Engenharia de São Carlos, Universidade de São Paulo, 2003.

[8] FERNANDES, G. R., SOUZA NETO, E.A. "Self-consistent linearization of non- linear BEM formulations with quadratic convergence”, Computational Mechanics, v. 52, pp. 1125-1139, 2013.

[9] FERNANDES, G. R, VENTURINI, W.S. "Non-linear boundary element analysis of floor slabs reinforced with rectangular beams”, Eng Anal Bound Elem, v.31, pp.721-737, 2007. 
[10] FERNANDES, G. R, VENTURINI W,S. "Non-linear boundary element analysis of plates applied to concrete slabs”, Eng Anal Bound Elem, v.26, pp.169-181, 2002

[11] OLIVEIRA NETO, L, PAIVA, J.B. “A special BEM for elastostatic analysis of building floor slabs on columns”, Comput Struct, v.81, n.6, pp. 359-372, 2003.

[12] RIBEIRO, G. O. Sobre a formulação do método dos elementos de contorno de placas usando as hipóteses de Reissner, Tese de D.Sc., Escola de Engenharia de São Carlos -USP, 1992.

[13] SIMO, J.C, TAYLOR, R.L. "Consistent tangent operators for rateindependent elastoplasticity”, Comput Methods Appl Mech Eng, v.48, pp.101-118, 1985.

[14] WAIDEMAM, L, VENTURINI, W.S. "A boundary element formulation for analysis of elastoplastic plates with geometrical nonlinearity”, Comput Mech, v. 45, pp. 335-347, 2010. 